\title{
SECOND LINE DRUGS RESISTANT MYCOBACTERIUM TUBERCULOSIS IN MULTI- DRUG RESISTANT TUBERCULOSIS PATIENTS
}

\author{
P. Tuladhar, D. K. Khadka, M. R. Banjara and R. Tuladhar
}

Journal of Institute of Science and Technology

Volume 22, Issue 2, January 2018

ISSN: 2469-9062 (print), 2467-9240 (e)

Editors:

Prof. Dr. Kumar Sapkota

Prof. Dr. Armila Rajbhandari

Assoc. Prof. Dr. Gopi Chandra Kaphle

Mrs. Reshma Tuladhar

JIST, 22 (2): 168-174 (2018)

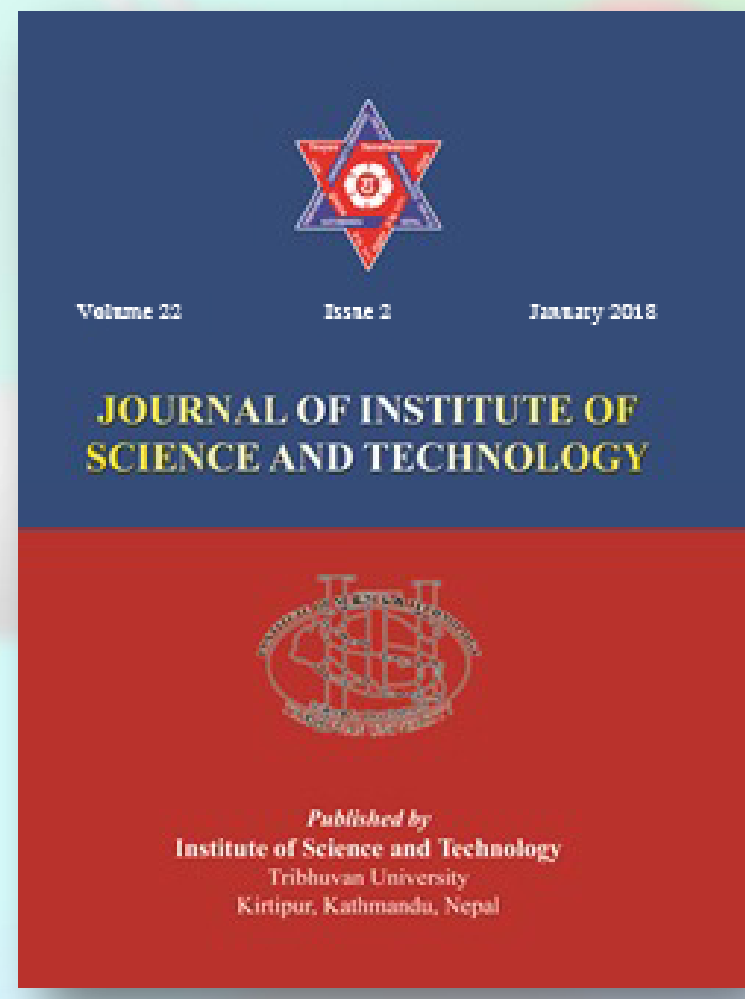

Published by:

Institute of Science and Technology

Tribhuvan University

Kirtipur, Kathmandu, Nepal 


\title{
SECOND LINE DRUGS RESISTANT MYCOBACTERIUM TUBERCULOSIS IN MULTI-DRUG RESISTANT TUBERCULOSIS PATIENTS
}

\author{
Pranita Tuladhar ${ }^{1}$, Dhruba Kumar Khadka ${ }^{2}$, Megha Raj Banjara ${ }^{1}$, Reshma Tuladhar, ${ }^{1, *}$ \\ ${ }^{1}$ Central of Department Microbiology, Tribhuvan University, Kirtipur, Kathmandu, Nepal \\ ${ }^{2}$ National Tuberculosis Centre, Thimi, Bhaktapur, Nepal \\ ${ }^{*}$ Corresponding E-mail: reshma.tuladhar@microbiotu.edu.np
}

Received: 22 November, 2017; Revised: 5 December, 2018; Accepted: 15 December, 2018

\begin{abstract}
With an increase in Multi-drug resistant tuberculosis (MDR-TB), there is a need of second line drug susceptibility test that helps in early diagnosis and minimize the risk of other powerful drug resistant Mycobacterium tuberculosis. The aim of this study was to determine second line drugs (ofloxacin, kanamycin, capreomycin) resistance pattern in MDR-TB isolates and to determine the prevalence of preExtensively drug resistant tuberculosis (pre-XDR-TB) and XDR-TB in MDR-TB patients. The study was conducted from February to September 2015 at National Tuberculosis Centre, Thimi, Bhaktapur. MDR-TB (resistant to isoniazid and rifampicin) patients' sputum samples were processed by Modified Petroff's method. Out of 92 samples, 57 were found culture positive. Following the species identification of culture positive MDR-TB isolates, second line drug susceptibility test was performed by conventional proportion method. Of 57 MDR-TB isolates, 22 (38.59\%) showed resistance to ofloxacin (Ofx), 9 (15.79\%) to capreomycin $(\mathrm{Cm})$ and $9(15.79 \%)$ to kanamycin $(\mathrm{Km})$. One XDR-TB $(1.8 \%)$ resistant to all drugs was detected. Of the remaining, 21(36.8\%) were resistant to ofloxacin only and $8(15.4 \%)$ were resistant to two drugs i.e. 29 (50.9\%) were pre-XDR-TB. The prevalence of pre-XDR-TB and XDR-TB was found to be $50.88 \%$ and $1.75 \%$ respectively. The resistance pattern of second line anti-tuberculosis drugs showed higher ofloxacin resistance in MDR-TB patients. In a nutshell, MDR-TB cases need urgent and timely susceptibility report for second line anti-tuberculosis drugs to help the clinicians start proper drug combinations to treat MDR-TB patients.
\end{abstract}

Keywords: XDR-TB, MDR-TB, Isoniazid, Rifampicin, Fluoroquinolones.

\section{INTRODUCTION}

Regardless of the effective and affordable discovery of chemotherapy, tuberculosis has remained a major cause of death worldwide (Glaziou et al., 2015). With the wide use of standard short course regimen, multidrug resistant tuberculosis (MDR-TB- defined as resistance to both isoniazid and rifampicin with or without resistance to other first-line drugs) incidence has increased. Significantly, high rates of MDR-TB have been observed in previously treated TB patients as well as new TB patients (Kim, 2005).

In 2013, WHO reported that 5\% of TB cases globally were multidrug resistant tuberculosis (MDR-TB). Drug resistance surveillance data show that an estimated 480,000 people developed MDR-TB in
2013 and 210,000 people died (WHO, 2014). The inappropriate use of second line anti-tuberculosis drugs in MDR-TB patients will lead to amplification of resistance and development of pre-extensively drug resistant tuberculosis (pre-XDR-TB- defined as MDR with resistance to fluoroquinolones or any of the three injectables second- line drugs (amikacin, kanamycin and capreomycin) and Extensively drug resistant tuberculosis (XDR-TB), defined as MDR with resistance to fluoroquinolones and any of the three injectables second- line drugs (amikacin, kanamycin and capreomycin) (WHO, 2012). On average, $9 \%$ of people with MDR-TB have XDR-TB (WHO, 2014).

TB is ranked as the sixth leading cause of death among top 20 causes of death in Nepal (NTP, 
2014). The latest drug resistance survey (2011-12) results have shown that the levels of drug resistance are high, with nearly $9.3 \%$ of new patient resistant to at least one drug. Levels of MDR-TB are low among new cases $(2.2 \%)$ than among retreatment cases $(15.4 \%)$. The high level of resistance to fluoroquinolones $(3.6 \%)$ in Nepal leads to a heavy burden of pre-XDR and XDR-TB among MDR-TB patients (XDR-TB is estimated at 8\%) (NTP, 2014). Few laboratories in Nepal have capacity to perform first-line and second-line drug susceptibility test for Mycobacterium tuberculosis. This study was aimed to determine the second-line anti-tuberculosis drug resistance pattern in MDR-TB patients as well as the prevalence rate of pre-XDR-TB and XDR-TB patients in Nepal.

\section{MATERIALS AND METHODS}

A descriptive study was carried out at National Tuberculosis Centre (NTC), Thimi, Bhaktapur, Nepal from February to August 2015. Total 92 sputum samples collected from MDR-TB patients attending NTC were included in this study. Whereas, non MDR-TB clinical isolates, other specimens like blood, CSF, urine, saliva and pus swab, and improperly collected samples like were excluded from the study.

\section{Specimen collection, microscopic examination and culture}

The sputum samples of MDR-TB patients were collected at NTC as per the standard protocol (WHO, 1998). The desired sputum samples were processed by Modified Petroff's method for performing Ziehl-Neelson (ZN) staining The grading of $\mathrm{ZN}$ staining was done as per the protocol. The samples were cultured in LowensteinJenson (LJ) medium following the WHO protocol. The cultures were incubated at $37^{\circ} \mathrm{C}$ for 8 weeks with weekly observation for growth. Bacterial colonies were identified as $M$. tuberculosis by colony characteristics and biochemical tests such as catalase, nitrate reduction test and paranitrobenzoic acid test (WHO, 1998).

\section{Drug susceptibility tests}

All confirmed culture positive MDR-TB isolates were subjected for second line drug susceptibility test by proportion method as per WHO standard protocol (WHO, 2001). Second-line anti-tuberculosis antibiotics ofloxacin (Ofx), kanamycin $(\mathrm{Km})$ and capreomycin $(\mathrm{Cm})$ from Sigma was used in the experiment. Cncentrations for ofloxacin, kanamycin and capreomycin were $1,20,10 \mu \mathrm{g} / \mathrm{ml}$ respectively. The antibiotics free control media were prepared simultaneously. A standard $1 \mathrm{mg} / \mathrm{ml}$ bacillary suspension (McFarland No.1) was prepared and used to compare the density of neat suspension which was further diluted in sterile distilled water to give $10^{-2}$ and $10^{-4}$ dilutions. Anti-tuberculosis antibiotics incorporated media and a control medium of plain LJ media were inoculated with one loop full of bacillary suspension from $10^{-2}$ dilution. Similarly, a loop full of bacillary suspension from $10^{-4}$ dilution was inoculated in each of all the antibiotics incorporated media and two controls of plain LJ media. The slopes were incubated at $37^{\circ} \mathrm{C}$ and the results were noted on the $4^{\text {th }}$ and $8^{\text {th }}$ weeks. A standard strain $\mathrm{H}_{37} \mathrm{R}_{\mathrm{v}}$ was included with every batch to check for antibiotics concentration. Any colony growing on antibiotics containing medium inoculated with the $10^{-2}$ dilution that equals or more the number of colonies growing on the control medium inoculated with the $10^{-4}$ dilution represents $1 \%$ or more of the test population. If the growth on the antibiotics containing media is $1 \%$ or more, then it was interpreted as resistant otherwise susceptible.

\section{Data analysis}

Frequency and percentages were calculated for qualitative variables like gender and drug sensitivity and using Microsoft Office Excel 2007. SPSS version 16 was used to calculate chi-square to determine the association between gender and second-line anti-tuberculosis drugs (ofloxacin, kanamycin and capreomycin). The prevalence of XDR-TB and pre-XDR-TB among MDR-TB patients was calculated using the formula.

\section{RESULTS}

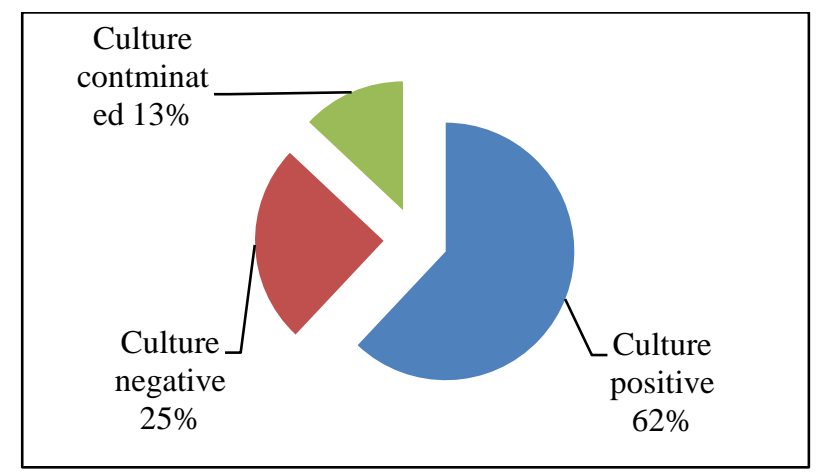

Fig. 1. Primary culture of MDR-TB patients.

In this study, out of 92 samples, 23 (25\%) samples did not show growth in primary culture, 12 $(13.04 \%)$ samples were found to be contaminated and $57(61.96 \%)$ samples showed growth in 
primary culture (Figure 1). Total 57 confirmed MDR-TB isolates were tested for anti-TB susceptibility pattern for second-line drugs (SLDs). Among 57 confirmed MDR-TB isolates, 30 (52.63\%) MDR-TB isolates were from male patients and 27 (47.37\%) from female patients; male to female ratio was found to be 10:9.
Out of 57 MDR-TB isolates, 27 (47.4\%) were sensitive and $30(52.6 \%)$ resistant to second line anti-tubercular drugs. Among these, 1 (1.8\%) was resistant to all three drugs i.e. XDR-TB, 21 (36.8\%) were resistance to ofloxacin only and $8(15.4 \%)$ were resistance to two drugs i.e. 29 (50.9\%) were pre-XDR-TB (Table 1).

Table 1: Resistance pattern of second-line anti-TB drugs of MDR-TB isolates on LJ media by proportion method.

\begin{tabular}{|c|c|c|c|}
\hline Resistance profile & Antibiotics & $\begin{array}{l}\text { Number of MDR- } \\
\text { TB isolates }\end{array}$ & Percentage \\
\hline \multirow[t]{3}{*}{ Resistance to 1 drug } & Resistance to ofloxacin & 21 & $36.8 \%$ \\
\hline & Resistance to capreomycin & 0 & $0 \%$ \\
\hline & Resistance to kanamycin & 0 & $0 \%$ \\
\hline \multirow[t]{3}{*}{ Resistance to 2 drugs } & $\begin{array}{l}\text { Resistance to ofloxacin and } \\
\text { capreomycin }\end{array}$ & 0 & $0 \%$ \\
\hline & $\begin{array}{l}\text { Resistance to ofloxacin and } \\
\text { kanamycin }\end{array}$ & 0 & $0 \%$ \\
\hline & $\begin{array}{l}\text { Resistance to capreomycin and } \\
\text { kanamycin }\end{array}$ & 8 & $14 \%$ \\
\hline \multirow[t]{2}{*}{ Resistance to 3 drugs } & $\begin{array}{l}\text { Resistance to ofloxacin, } \\
\text { capreomycin and kanamycin }\end{array}$ & 1 & $1.8 \%$ \\
\hline & Susceptible & 27 & $47.4 \%$ \\
\hline
\end{tabular}

Of 57 MDR-TB isolates, $22(38.59 \%)$ showed resistance to ofloxacin (Ofx) (MIC- $1 \mu \mathrm{g} / \mathrm{ml}), 9$ $(15.79 \%)$ to capreomycin $(\mathrm{Cm})(\mathrm{MIC}-10 \mu \mathrm{g} / \mathrm{ml})$ and $9(15.79 \%)$ to kanamycin (Km) (MIC-
$20 \mu \mathrm{g} / \mathrm{ml}$ ) (figure 2). One XDR-TB was recovered from a male of 22 yrs. Out of 29 pre-XDR-TB cases, 14 were recovered from male and 15 from female.

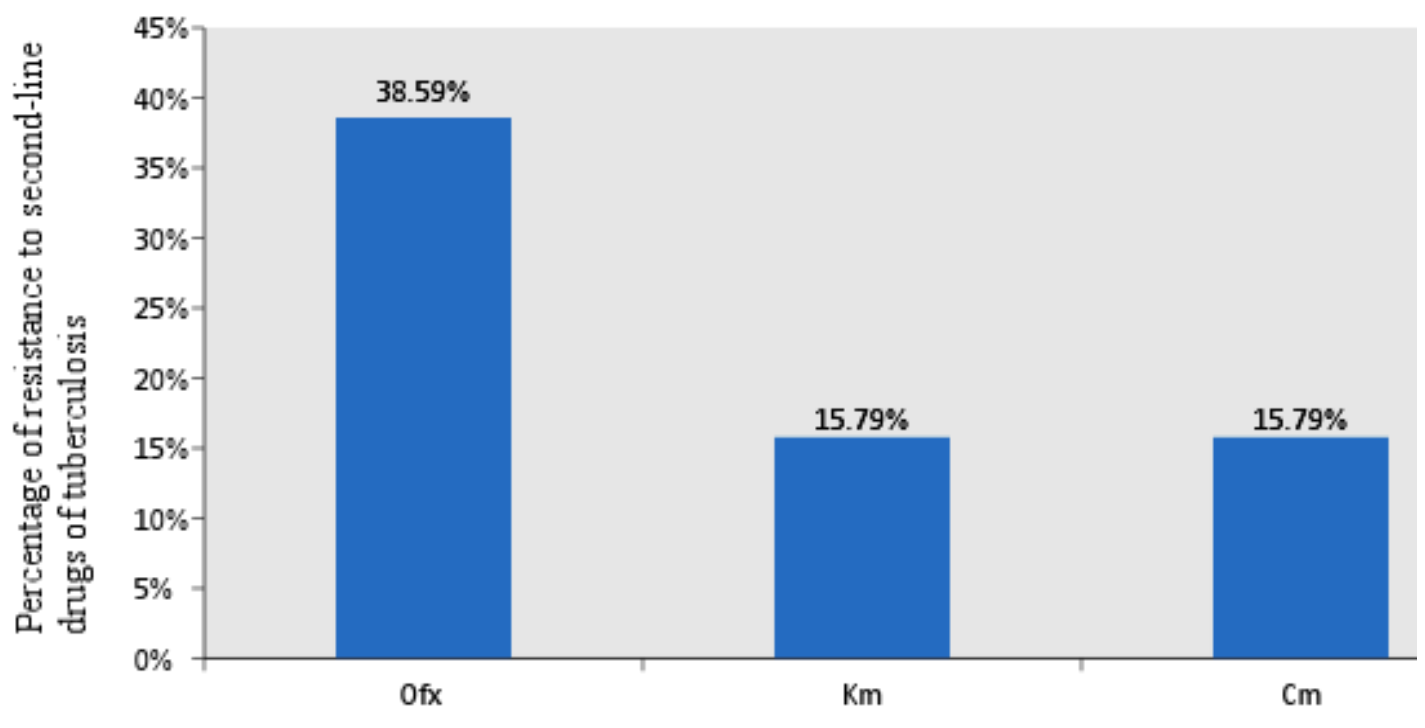

Second-line drugs used for second line drug susceptibility test

Fig. 2. Resistance pattern of MDR-TB isolates against second-line anti-TB drugs (n=57). 
Statistically, there is no significant association between gender and ofloxacin $(\mathrm{p}=0.629)$, gender and kanamycin $(\mathrm{p}=0.114)$ and gender and capreomycin $(\mathrm{p}=0.114)$. Out of 29 pre-XDR-TB cases, the highest number of pre-XDR-TB cases i.e. $10(34.6 \%)$ were observed in the age group of $11-$
20 years followed by $9(31 \%)$ in the age group of $21-30$ years, $3(10.3 \%)$ in $31-40$ years, $3(10.3 \%)$ in 41-50 years, $2(6.9 \%)$ in 51-60 years and $2(6.9 \%)$ in 61-70 years (Table 2). The prevalence of preXDR-TB and XDR-TB was calculated and found to be $50.88 \%$ and $1.75 \%$ respectively.

Table 2: Distribution of pre-XDR-TB patients in different age groups.

\begin{tabular}{|c|c|c|c|c|c|c|c|}
\hline S.N & Age group(years) & Male & \% of male & Female & \% of female & Total & Total \% \\
\hline 1 & $0-10$ & 0 & 0 & 0 & 0 & 0 & 0 \\
\hline 2 & $11-20$ & 3 & 5.3 & 7 & 12.3 & 10 & 34.6 \\
\hline 3 & $21-30$ & 5 & 8.8 & 4 & 7 & 9 & 31 \\
\hline 4 & $31-40$ & 2 & 3.5 & 1 & 1.8 & 3 & 10.3 \\
\hline 5 & $41-50$ & 2 & 3.5 & 1 & 1.8 & 3 & 10.3 \\
\hline 6 & $51-60$ & 1 & 1.8 & 1 & 1.8 & 2 & 6.9 \\
\hline 7 & $61-70$ & 1 & 1.8 & 1 & 1.8 & 2 & 6.9 \\
\hline 8 & $71-80$ & 0 & 0 & 0 & 0 & 0 & 0 \\
\hline & Total & 14 & & 15 & & 29 & 100 \\
\hline
\end{tabular}

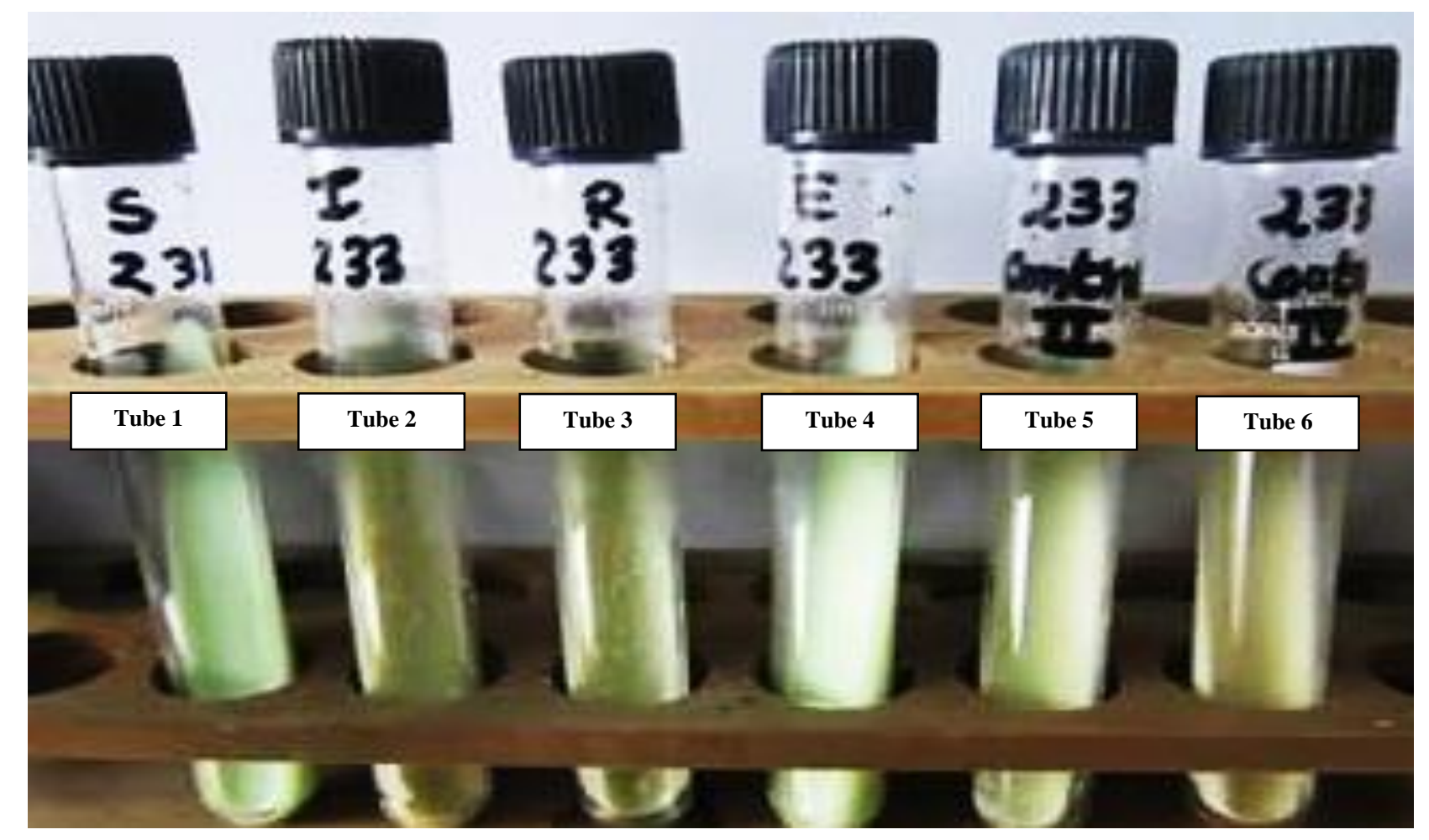

Fig. 3. First-line drug susceptibility test showing M. tuberculosis resistant to isoniazid and rifampicin

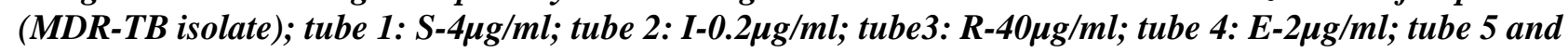
6: Controls for dilution II and IV. 


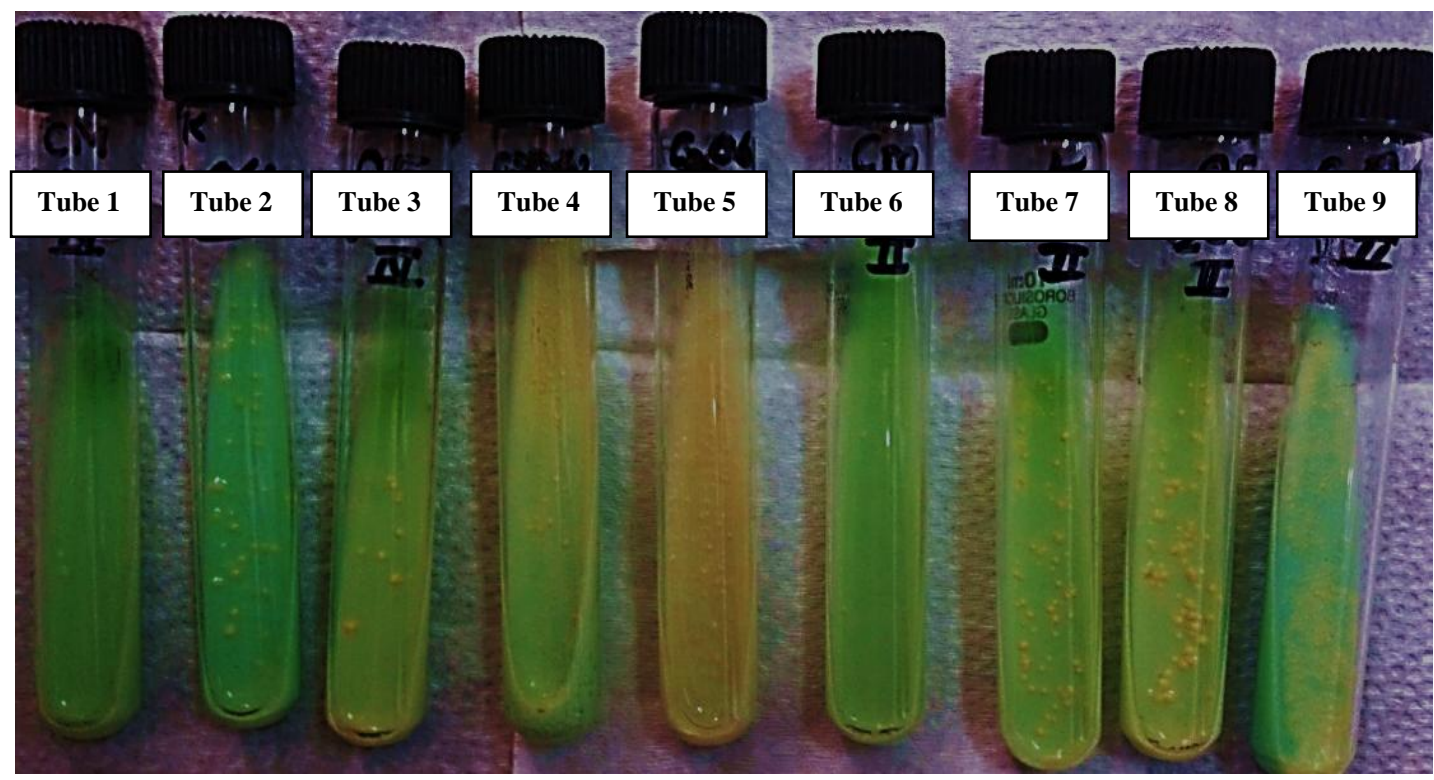

Fig. 4. Second line drug susceptibility test showing M. tuberculosis resistant to ofloxacin(Ofx) and kanamycin(Km) (XDR-TB strain). Tube 4 and 5: Controls for dilution IV; tube 9: Control for dilution II; tube 3 and 8: $O f x-1 \mu \mathrm{g} / \mathrm{ml}$ for dilution IV and II; tube 2 and 7: Km-20 $\mu \mathrm{g} / \mathrm{ml}$ of dilution IV and II; tube 1 and 6: $\mathrm{Cm}-10 \mu \mathrm{g} / \mathrm{ml}$ for dilution IV and II.

\section{DISCUSSION}

In the countries with high TB prevalence, MDR-TB is one of the growing problems which have led to increase in other drug resistant cases of TB such as pre-XDR-TB and XDR-TB cases. For this reason, MDR-TB cases should be timely monitored. A significant part of monitoring process of TB resistance to drugs is drug susceptibility testing of M. tuberculosis (WHO, 1997). This requires laboratories with facilities of drug susceptibility to be established at different places.

Several methods are used for processing of samples. As a general rule, a contamination rate of $2-3 \%$ is acceptable for fresh specimen and $5-10 \%$ if specimens (especially sputum) are delayed to the laboratory (SAARC, 2009). From this study, the contamination rate of the sputum culture was found to be high i.e. $13 \%$ than the acceptable range. The samples were delayed in receiving to the laboratory since more samples were brought from different TB centers of Nepal by courier in a sealed and refrigerated condition. Although the samples were kept in a refrigerated condition, many contaminating bacteria surviving in cold condition may overgrow in the culture media. This leads to low recovery i.e. low number of growth (positive cases). The processing time for the sputum may not have been enough to kill the contaminating bacteria for some of the sputum samples.
In Nepal, National Tuberculosis Centre (NTC) and GENETUP are the only two laboratories that have the facility of conducting drug susceptibility test for both first-line and second-line anti-tuberculosis drugs. This study was conducted at NTC and was carried out mainly on three second line antituberculosis drugs i.e. ofloxacin, kanamycin and capreomycin which belong to fluoroquinolones, aminoglycosides and polypeptides classes of the antibiotic respectively.

A study conducted in Pakistan by Mirza et al. (2015) showed $87 \%$ of MDR-TB isolates were resistant to capreomycin and 53\% resistant to ofloxacin as compare to the finding of $14 \%$ and $36.8 \%$ respectively. This finding is in contrast to reports from Pakistan where resistance to capreomycin is much higher as compare to ofloxacin. Another study conducted by Olusoji et al. (2013) found that $80 \%$ of MDR-TB isolates were resistant to ofloxacin and 20\% to kanamycin. This study is also in contrast to this finding of $36.8 \%$ of MDR-TB isolates resistant to ofloxacin and $14 \%$ to kanamycin respectively. It showed high resistance towards ofloxacin as compare to this finding.

In 2015, Patel et al. found 18 (36\%) of MDR-TB isolates were resistant to ethionamide, 13 (28\%) to Dcycloserine, $11(22 \%)$ to ciprofloxacin, 7 (14\%) to kanamycin, 6 (12\%) to para-aminosalicylic acid and 5 $(10 \%)$ to amikacin. This finding regarding kanamycin 
is in concordance with our study where $14 \%$ of MDRTB showed resistance to kanamycin. A similar study conducted by Ghafoor et al. (2015) found that out of 100 MDR-TB isolates, $62 \%$ were from male patients and $38 \%$ from female patients. $97 \%$ were sensitive to amikacin, $53 \%$ to ofloxacin, $87 \%$ to capreomycin and $87 \%$ were sensitive to ethionamide. The result regarding capreomycin is in concordance with this study which showed $85.96 \%$ were sensitive to capreomycin. Whereas, it showed less sensitivity to ofloxacin compared to this study.

In a study conducted by Paramasivian et al. (2010), out of 1498 MDR-TB cases, 32.7\% were resistant to ethionamide, $16.4 \%$ to ofloxacin and $11.3 \%$ to kanamycin; $4.9 \%$ were extensively drug-resistant TB (XDR-TB). It showed less resistance towards ofloxacin and kanamycin than this study however the percentage of XDR-TB were high as compare to this study of $1.75 \%$ XDR-TB.

The strongest risk factor for resistance to secondline drugs was previous treatment with these drugs consistently, which increased the risk of XDR tuberculosis by more than four times. Fluoroquinolone resistance and XDR tuberculosis were more frequent in women than in men (Dalton et al., 2012). In this study, the frequency of fluoroquinolone resistance among men and women was nearly similar. Fourteen pre-XDR-TB cases were from male patients and fifteen from female patients. This result is in concordance with the study done by Dalton et al. (2012).

Majority of the pre-XDR-TB bacilli are resistant to ofloxacin than other two classes of drugs. This may be because of the over the counter availability and unregulated use of fluoroquinolones (NTP, 2014). Fluoroquinolones are susceptible to indiscriminate use for bacterial infections (Mirza et al., 2015). Patients who have been exposed to fluoroquinolones prior to diagnosis of $\mathrm{TB}$ are at the higher risk of infection from fluoroquinolones resistant $\mathrm{TB}$ than patients with $\mathrm{TB}$ who have no previous exposure to this group of drugs (Mirza et al., 2015). Another report showed that even a short duration of monotherapy with fluoroquinolones can quickly result in acquired resistance in M. tuberculosis (Patel et al., 2015). Prescription of an inappropriate drug for active TB in the private health sector and over-the-counter availability of fluoroquinolones should be prohibited (Dalal et al., 2015). All patients at the risk of suffering from active TB on account of drug-resistant strain need to have second-line antiTB drugs during the initial phase (Dalal et al.,
2015). As stated in NTP, to reduce mortality due to $\mathrm{XDR}-\mathrm{TB}$, it is recommended to perform DST for second-line drugs on all MDR-TB cases at the start of the treatment. To fulfill this, Nepal has to strengthen its capacity to perform second-line antiTB drug susceptibility testing, either by culture (solid/liquid) or molecular biology (Line Probe Assay).

The ultimate strategy to control multidrug resistant tuberculosis is to implement comprehensive approach incorporating treatment of multidrug resistant tuberculosis based upon principles closely related to those of its general DOTS strategy for TB control: sustained political commitment; a rational case-finding strategy including accurate, timely diagnosis through quality assured culture and DST; appropriate treatment strategies that use second-line drugs under proper case management conditions; uninterrupted supply of quality-assured antituberculosis drugs; standardized recording and reporting system (Marahatta, 2010).

This study had no access to genetic data for MDRTB population, which could have complemented the description of the circulating strains. Despite its limitations, this study adds important data and insights into the description of the drug-resistant tuberculosis epidemic in Nepal.

It is important to have accurate detection of drug resistance along with quality assured DST against first and second-line anti-TB drugs for subsequent decisions to promptly manage TB and MDR-TB patients. Though the results will be beneficial to clinicians for prescribing second-line anti-TB drugs to MDR-TB patients, more health centers are required to establish DST facilities. The TB drug resistance patterns in Nepal highlight the need for individualized drug regimens, designed on the basis of full drug susceptibility results to first- and second-line anti-TB drugs.

\section{CONCLUSION}

Hence we conclude that the multidrug resistant TB cases need urgent and timely susceptibility report for second line anti-tuberculosis drugs to help the clinicians start proper drug combinations to treat MDR-TB patients. More detailed and populationbased studies are required to know the burden of XDR-TB strains in community. XDR-TB is throwing an open challenge to clinicians and policy makers as M. tuberculosis is growing immortal and devastating as the cases of All drug resistant TB (ADR-TB) have arisen which can't be treated with anti-tuberculosis drug known up to now. 


\section{ACKNOWLEDGEMENT}

We would like to thank all the staff of National Tuberculosis Centre and SAARC Tuberculosis and HIV/AIDS Centre, Thimi, Bhaktapur, Nepal for their constant help during this study. We are also thankful to all patients who provided the samples.

\section{REFERENCES}

Dalal, A.; Pawaskar, A.; Das, M.; Desai, R.; Prabhudesai, P.; Chhajed, P. et al. (2015). Resistance patterns among Multidrug Resistant Tuberculosis Patients in Greater Metropolitan Mumbai: Trends over Time. PLos One, 10: e0116798.

Dalton, T.; Cegielski, P.; Akksilp, S.; Asencios, L.; Campos, J. C.; Cho, S. N. et al. (2012). Prevalence of and risk factors for resistance to second-line drugs in people with multidrug-resistant tuberculosis in eight countries: a prospective cohort study. The Lancet, 380:1406-1417.

Ghafoor, T.; Ikram, A.; Abbasi, S. A.; Zaman, G.; Ayyub, M.; Palamino, J. C. et al. (2015). Sensitivity Pattern of Second Line AntiTuberculosis Drugs against Clinical Isolates of Multidrug Resistant Mycobacterium tuberculosis. Journal of College of Physicians and Surgeons Pakistan, 25: 250-253.

Glaziou, P.; Sismanidis, C.; Floyd, K. and Mario, Reviglione (2015). Global Epidemiology of Tuberculosis. Cold Spring Harbor Perspectives in Medicine, 5 (2): a017798.

Kim, S. J. (2005). Drug susceptibility testing in tuberculosis: methods and reliability of results. The European Respiratory Journal, 25:564-569.

Marahatta, S. B. (2010). Multi-drug resistant tuberculosis burden and risk factors: an update. Kathmandu University Medical Journal (KUMJ), 8:116-125.

Mirza, I. A.; Khan, F. A.; Khan, K. A.; Satti, L.; Ghafoor, T. and Fayyaz, M. (2015). Extensively and Pre-extensively Drug Resistant Tuberculosis in Clinical Isolates of Multi-drug Resistant Tuberculosis Using Classical Second Line Drugs (Levofloxacin and Amikacin). Journal of College of Physicians and Surgeons Pakistan, 25 (5): 337-341.
National Tuberculosis Programme (2014). Annual report 2070/2071 Nepal. National Tuberculosis Centre, Thimi, Bhaktapur, Nepal.

Olusoji, D.; Eltayeb, O.; Olanrewaju, O. and Olapade, G. D. (2013). Pre-Extensively Drug Resistance Tuberculosis (Pre-XDR-TB) among MDR-TB patents in Nigeria. Global Advanced Research Journal of Microbiology, 2: 022-025.

Paramasivan, C. N.; Rehman, F.; Wares, F.; Sundar, M. N.; Sundar, S.; Devi, S. et al. (2010). First- and second-line drug resistance patterns among previously treated tuberculosis patients in India. International Journal of Tuberculosis and Lung Disease, 14: 243-246.

Patel, S. M.; Patel, M. H.; Soni, S. T. and Vegad, M. M. (2015). Second-line drug resistance patterns among patients with multidrug-resistant tuberculosis of Gujarat, western India. International Journal of Medical Science and Public Health, 4: 639-641.

SAARC (2009). Training manual for Mycobacterium tuberculosis Culture and Drug Susceptibility Testing. Central TB division. New Delhi.

WHO/IUATLD (1997). Global Project on AntiTuberculosis Drug Resistance Surveillance 1994-1997. Anti-tuberculosis drug resistance in the world. Geneva: WHO Tuberculosis Global Programme.

WHO (1998). Laboratory services in tuberculosis control, Part III: Culture. Geneva: World Health Organization.

WHO (2001). Guidelines for drug susceptibility testing for second-line anti-tuberculosis drugs for DOTS-PLUS. Communicable Diseases. WHO/CDS/TB/2001.288.

WHO (2012). Global tuberculosis report 2012. http://apps.who.int/iris/bitstream/10665/7593 8/1/9789241564502_eng.pdf.

WHO (2014). Global TB report 2014. WHO, Geneva. 